\title{
Evaluación del dolor con variaciones del videopupilómetro, usando analgesia con tapentadol en Terapia Intensiva Adultos
}

\author{
Nancy Verónica Alva Arroyo, ${ }^{1}$ José Manuel Athíe García, ${ }^{2}$ Enrique Monares Zepeda, ${ }^{3}$ \\ Diego Jaime Villalón ${ }^{4}$
}

\section{Resumen}

En la Unidad de Terapia Intensiva (UTI) la evaluación precisa del dolor es esencial; se informó que 35-55\% de las enfermeras subestiman el dolor del paciente. El objetivo principal de este estudio es observar si la variación del índice de dolor pupilar determina un umbral pronóstico de la eficacia analgésica con el uso de tapentadol en pacientes ingresados a terapia intensiva y a su egreso. Las mediciones se llevaron a cabo por personal médico, con el uso de AlgiScan (videopupilómetro) y escala visual análoga; se incluyeron pacientes adultos ingresados a terapia intensiva que requerían infusiones con opioides, por más de 48 horas. Es un estudio a manera de ensayo clínico controlado, donde se administró tapentadol en un grupo y en un grupo control analgesia estándar. Se valoró videopupilómetro a las cuatro y a las 12 horas de administrar tapentadol, hasta su egreso. Sobre un total de 40 pacientes, 19 recibieron tapentadol y 21 analgesia estándar. Al comparar entre grupos el resultado numérico obtenido por videopupilómetro, se encontró una diferencia estadísticamente significativa $(p<0.001)$, con una media de 11 (grupo de tapentadol) contra una media de 19 (grupo de otras analgesias), siendo claras las calificaciones de menor dolor en el grupo de tapentadol.

Palabras clave: Evaluación de dolor en UTI, videopupilómetro, tapentadol.

\begin{abstract}
Summary
Precise pain assesment within the Intensive Care Unit (ICU) is essential. It has been noted that approximately $33-35 \%$ of nursing staff consistently underestimate their patients' pain. Our study's main objective is to observe if the variations of the pupillary pain index determine a prognostic threshold during tapentadol use, when in the Intensive Care Unit and after discharge. Pupillary measurements were done by local medical personnel by means of the AlgiScan device (videopupillometer) and the visual analogue scale. Adult patients being admitted to the ICU and whose time requirements of opioid infusions were predicted to be longer than 48 hours were considered for inclusion. Designed as a controlled clinical trial, tapentadol use in a group was compared to standard analgesia as a control group. Videopupillometry was performed at four hours post tapentadol administration, then every 12 hours until medical discharge from the ICU. A total of 40 patients were included, 19 in the tapentadol group and 21 in the standard analgesia group. When compared between groups, the numerical variable obtained by pupillometry was found to be different with statistical significance $(p<0.01)$, with a mean of 11 for the tapentadol group and a mean of 19 in the standard analgesia group. A lower pain score in the tapentadol group when compared with standard analgesia was made evident in this study.
\end{abstract}

Key words: Treatment of pain un ICU, AlgiScan, tapentadol.

\footnotetext{
${ }^{1}$ Médico Anestesiólogo y Medicina Crítica, Hospital Ángeles Mocel, Universidad La Salle.

2 Director Médico, Hospital Ángeles Mocel.

3 Jefe de Terapia Intensiva, Hospital San Ángel Inn Universidad.

${ }^{4}$ Infectología, Hospital San Ángel Inn Universidad.
}

Correspondencia:

Nancy Verónica Alva Arroyo

Correo electrónico: nan_ve@hotmail.com

Aceptado: 27-09-2016.

Este artículo puede ser consultado en versión completa en http://www.medigraphic.com/actamedica 


\section{INTRODUCCIÓN}

La Unidad de Terapia Intensiva (UTI) típica no es un lugar tranquilo y reposado. Aun en la UTI más moderna construida para proporcionar un ambiente más humano, los pacientes están inmersos en un torbellino de ruido, equipos, personas, actividad... Se viola el espacio y la autonomía personales. Los pacientes a menudo están confinados en una cama debido a que están conectados a equipos para el soporte vital. Es posible que el paciente en estado crítico presente una enfermedad grave, dolor, ansiedad, miedo, incapacidad para comunicarse en forma adecuada con el personal de la UTI; esto puede conducir al desarrollo del delirio. La parte más importante en el manejo del dolor es identificar la causa. ${ }^{1,2}$

La evaluación precisa del dolor en el paciente es esencial; se ha informado que 35 a 55\% de las enfermeras lo subestiman. ${ }^{2}$ Sin embargo, muchos pacientes críticamente enfermos son incapaces de comunicarse de manera efectiva debido al deterioro cognitivo, sedación, parálisis o ventilación mecánica. ${ }^{2}$ Cuando los pacientes no pueden expresarse, hay indicadores observables tanto fisiológicos y de comportamiento, que se han utilizado para evaluar el dolor en esta población. Es importante mencionar que un dolor puede ser leve para un paciente pero agónico para otro; sin embargo, los medicamentos utilizados para aliviar el dolor y la ansiedad inducen el sueño, son potencialmente peligrosos y tienen efectos secundarios. En la literatura se ha descrito una variedad de escalas de sedación, pero una de las más utilizadas es la de Ramsay, RASS (Richmond Agitation Scale Sedation)., ${ }^{3,4}$

\section{Antecedentes}

Las evaluaciones del dolor en la UTI, utilizando los instrumentos de evaluación son válidas pero poco fiables; éstas deberían proporcionar la base para el tratamiento del dolor en los pacientes críticos. ${ }^{5}$

La NPAT (herramienta de evaluación del dolor no verbal) hace una fuerte recomendación para la realización de evaluaciones de dolor de rutina en todos los pacientes ingresados en la UTI. El estándar de oro en la evaluación de dolor es que el paciente lo describa o caracterice; el NRS (escala numérica) es la escala de 0-10 (0 dolor nulo o mínimo, 10 el más fuerte que haya experimentado). La escala de dolor no verbal para el adulto (NVPS) está basada en gestos faciales, actividad de pies (movimientos), llanto, consolabilidad. Tanto la NPAT y la NVPS valoran expresión facial, actividad, vigilancia y constantes fisiológicas como frecuencia cardiaca, presión arterial, frecuencia respiratoria; se clasifican en dolor leve o severo; y la NVPS incluye indicadores autonómicos tales como midriasis, diaforesis, rubor, palidez... Cada apartado se clasifica en 0 a 2, con una puntuación total entre 0 (sin dolor) y 10 (dolor máximo) (Cuadro 1). ${ }^{6}$ Las guías para el manejo de dolor, agitación y delirio, recomiendan usar varias herramientas del comportamiento de noche, utilizando para detectar y evaluar el dolor la profundidad de la sedación y el grado de agitación, así como la detección temprana del delirio. ${ }^{7}$

Otra escala es la CPOT (herramienta para observar el dolor en cuidados críticos). Las escalas CPOT, NVPS y BPS demuestran una buena fiabilidad entre los evaluadores, tanto en pacientes de la UTI intubados como no intubados, cuando éstos son incapaces de comunicar el dolor. ${ }^{8}$

Es de gran importancia el control del dolor en UTI, debido a la alta incidencia de dolor crónico que se ha estudiado, y que presentan los pacientes que sobreviven a la enfermedad crítica, incluso después de seis meses y hasta un año de su egreso. ${ }^{9}$

El dolor severo y los eventos adversos graves son comunes y fuertemente asociados al mover pacientes en UTI para los procedimientos de enfermería. Al mejorar la calidad en el manejo del dolor se disminuyen eventos graves. La documentación cuidadosa del manejo del dolor durante la movilización en los procedimientos podría ser implementada como un indicador de la calidad de la salud en UTI. ${ }^{10}$

El videopupilómetro (AlgiScan) es una herramienta que mide el índice de dolor pupilar (PPI); varía de 0 a 10 y es una medida compuesta del reflejo del diámetro pupilar a los estímulos de dolor. Los puntajes más pequeños indican un aumento en la analgesia. ${ }^{1}$

La dilatación del reflejo pupilar (RDP) mostró su especificidad y relevancia en la medición del nivel de analgesia de los pacientes, tanto en Unidad de Cuidados Intensivos como en sala quirúrgica. El AlgiScan (videopupilómetro), cuantifica en segundos el reflejo pupilar al medir el diámetro de dilatación por estimulación eléctrica a diferentes intensidades; también ayuda a proporcionar el nivel adecuado de analgesia para todos los procedimientos médicos y quirúrgicos. ${ }^{1}$

El tapentadol es un analgésico sintético de acción central que combina actividad opioide y no opioide en una sola molécula. Los datos preclínicos sugieren que la eficacia analgésica de tapentadol se relaciona tanto con la actividad agonista opioide del receptor $\mu$ como con la inhibición de la recaptación de noradrenalina. Las concentraciones máximas de tapentadol se observan de tres a seis horas posteriores a la administración de tabletas de liberación prolongada (Retard). La sustancia se distribuye ampliamente en todo el cuerpo; su volumen de distribución es 540 \pm 98. La unión a proteínas en plasma es baja y alcanza aproximadamente $20 \%$. El metabolismo es extenso, se metaboliza alrededor de $97 \%$ del compuesto. La vía principal de metabolismo de tapentadol es la conjugación con ácido glucorónico para producir glucorónidos. Luego de la administración oral, aproximadamente $70 \%$ de la dosis 
se excreta en orina como formas conjugadas ( $55 \%$ como glucorónido y $15 \%$ como sulfato de tapentadol). Ninguno de los metabolitos contribuye a la actividad analgésica. ${ }^{11}$

Tapentadol es 18 veces menos potente que la morfina para unirse al receptor opioide $\mu$ humano y $2-3$ veces menos potente para producir analgesia en los modelos animales, lo cual es consistente con su mecanismo de acción combinada. En modelos preclínicos, la actividad analgésica debida a los agonistas opioides del receptor $\mu$ de tapentadol se puede modular mediante antagonistas selectivos de los receptores opioides $\mu$ (por ej., naloxona), mientras que la recaptación de la inhibición de noradrenalina es sensible a los moduladores de norepinefrina. ${ }^{12-14}$

Por tal motivo se llevó a cabo este estudio que consistió en obtener una medición objetiva del dolor durante el proceso de destete, al momento de la extubación y 48 horas posteriores, así como medir la analgesia durante su egreso de hospitalización.

¿La medición de la reactividad pupilar a la estimulación dolorosa por un pupilómetro automatizado determina la efectividad del tapentadol en el paciente crítico, bajo ventilación mecánica y postextubación?

El objetivo de este estudio es observar si la variación del índice de dolor a través del diámetro pupilar puede determinar un umbral pronóstico de la eficacia analgésica con el uso de tapentadol en pacientes ingresados a terapia intensiva y a su egreso.

\section{MATERIAL Y MÉTODOS}

Las mediciones se llevaron a cabo por personal médico adscrito de la terapia intensiva, con el uso de AlgiScan (videopupilómetro), después de la estimulación bilateral nociceptiva en pacientes con intubación orotraqueal, en proceso de destete de ventilación mecánica, y con disminución de más del $50 \%$ o suspensión de sedación y analgesia intravenosa.

El NeuroLight AlgiScan es un videopupilómetro portátil que permite medir la pupila de los pacientes rápida y fácilmente. Simultáneamente con la medición de la pupila, se pueden generar dos tipos de estimulación, fotoestimulación o estimulación eléctrica nerviosa, con el fin de evaluar la condición de analgesia, el estado hipnótico y el reflejo pupilar del paciente a la luz con respecto a las estimulaciones generadas, el uso clínico en anestesiología y el uso en terapia intensiva, en pacientes que requieran medición del dolor.

Se colocó el videopupilómetro portátil en cada ojo y se dio un disparo en modo PPI (La prueba PPI facilita la profundidad de la analgesia del paciente a través de la puntuación PPI, descrita más adelante). La duración de la prueba es de entre dos y 16 segundos. Durante toda la prueba, el usuario se mantuvo presionando el botón verde. El principio de funcionamiento consiste en aplicar al paciente una estimulación continua nociceptiva e ir aumentando en intensidad hasta una variación pupilar superior al 13\%. Cuando la variación del tamaño de pupila es superior al $13 \%$ se detiene la estimulación y el NeuroLight AlgiScan calcula la puntuación de PPI. Cada nivel de estimulación eléctrica tiene una duración de 1 seg; el aumento de estimulación en intensidad va desde 10 a 60 $\mathrm{mA}$ por pasos de $10 \mathrm{~mA}$ (Cuadro 2).

Los tiempos de medición fueron: a las cuatro horas de iniciarse la primera dosis de tapentadol tableta, y posterior-

Cuadro 1. Escala de dolor para adultos no verbal (adult nonverbal pain scale-NVPS). ${ }^{6}$

\begin{tabular}{|c|c|c|c|}
\hline Categorías & 0 & 1 & 2 \\
\hline Cara & $\begin{array}{l}\text { No expresión particular o } \\
\text { sonrisa }\end{array}$ & $\begin{array}{l}\text { Mueca ocasional, lagrimeo, } \\
\text { función del ceño, frente } \\
\text { arrugada }\end{array}$ & $\begin{array}{l}\text { Mueca frecuente, lagrimeo, } \\
\text { diaforesis, función del ceño, } \\
\text { frente arrugada }\end{array}$ \\
\hline Actividad (movimiento) & $\begin{array}{l}\text { No expresión, posición } \\
\text { normal }\end{array}$ & $\begin{array}{l}\text { Busca atención a través } \\
\text { del movimiento, lento o } \\
\text { cauteloso }\end{array}$ & $\begin{array}{l}\text { Inquieto, actividad excesiva } \\
\text { y/o reflejos de retirada }\end{array}$ \\
\hline Custodiando & $\begin{array}{l}\text { Sonidos bajos, sin mover } \\
\text { las manos por arriba del } \\
\text { cuerpo }\end{array}$ & $\begin{array}{l}\text { Rígido en las áreas del } \\
\text { cuerpo }\end{array}$ & Rígido, tenso \\
\hline $\begin{array}{l}\text { Signos vitales } \\
\text { (respiratorios) }\end{array}$ & $\begin{array}{l}\text { Equilibrio } \mathrm{FR} / \mathrm{SpO}_{2} \text { comple- } \\
\text { tamente con el ventilador }\end{array}$ & $\begin{array}{l}\text { FR > } 10 \text { basales, o } 5 \% \\
\text { menos } \mathrm{SpO}_{2} \text { moderada } \\
\text { asincronía con el ventilador }\end{array}$ & $\begin{array}{l}\mathrm{FR}>20 \text { basal, o } 10 \% \\
\text { menos de } \mathrm{SpO}_{2} \text { severa } \\
\text { asincronía con el ventilador }\end{array}$ \\
\hline
\end{tabular}

Tomado y modificado de: Stites M. Observational pain scales in critically ill adults. Crit Care Nurse. 2013; 33 (3): 68-78. 


\section{Cuadro 2. Escala de puntuación PPI. ${ }^{1}$}

\begin{tabular}{|c|c|c|}
\hline $\begin{array}{l}\text { Máxima intensidad } \\
\text { alcanzada por la } \\
\text { estimulación }(\mathrm{mA})\end{array}$ & $\begin{array}{c}\text { Puntuación } \\
\text { PPI }\end{array}$ & Nivel de reactividad de la pupila del paciente \\
\hline 10 & 9 & La dilatación de pupila sobrepasa el 13\% durante la estimulación de 10 mA \\
\hline 20 & 8 & La dilatación de pupila sobrepasa el 13\% durante la estimulación de 20 mA \\
\hline 30 & 7 & La dilatación de pupila sobrepasa el 13\% durante la estimulación de 30 mA \\
\hline 40 & 6 & La dilatación de pupila sobrepasa el 13\% durante la estimulación de $40 \mathrm{~mA}$ \\
\hline 50 & 5 & La dilatación de pupila sobrepasa el 13\% durante la estimulación de 50 mA \\
\hline 60 & 4 & La dilatación de pupila sobrepasa el 13\% durante la estimulación de 60 mA \\
\hline 60 & 3 & La dilatación de pupila sobrepasa el 13\% durante el segundo nivel de 60 mA \\
\hline $\begin{array}{l}60 \mathrm{~mA}(5 \% \\
<\text { dilatación de } \\
\text { pupila }<13 \%)\end{array}$ & 2 & Dilatación de pupila entre 5 y $13 \%$ durante la estimulación de tercer nivel de $60 \mathrm{~mA}$ \\
\hline $\begin{array}{l}60 \mathrm{~mA} \text { (dilatación de } \\
\text { pupila } \leq 5 \% \text { ) }\end{array}$ & 1 & La dilatación de pupila menor de $5 \%$ durante la última estimulación de $60 \mathrm{~mA}$ \\
\hline
\end{tabular}

Nota: Si la dilatación pupilar es superior al $20 \%$ durante la estimulación aumentamos la puntuación PPI un punto. Tomado de: www.idmed.fr. Manual NeuroLight AlgiScan. Versión 1.15 A5 - SP. 2013; 1-36.

mente cada 12 horas, con excepción de un paciente que refirió dolor (medido por signos vitales, por gesticulaciones o porque logró comunicarlo); en ese caso se dio dosis de rescate de tapentadol 50 mg, y se volvió a evaluar EVA a la hora y por videopupilómetro a las dos horas; en las primeras 24 horas se completó la dosis total, y se procuró vigilar efectos colaterales.

Se inició con dosis de 50 mg cada ocho horas (tableta Retard), si el peso era menor a 70 kg, y sin uso crónico de analgésicos de cualquier otro tipo o uso de benzodiacepinas.

Para pacientes con peso $>70 \mathrm{~kg}$ o uso crónico de analgésicos de cualquier tipo, se usaron de 100 a 200 mg cada ocho horas y con rescate si se requiere sin rebasar una dosis de $500 \mathrm{mg}$ al día.

Cabe señalar que en todos los casos, el uso del dispositivo en el paciente no debió exceder 15 segundos seguidos. El número de mediciones realizadas en el mismo paciente no debió exceder 10 por hora. Se dio un puntaje ya establecido en la evaluación del videopupilómetro.

\section{Diseño}

El presente estudio se realizó a manera de ensayo clínico controlado, realizando la intervención clínica en forma de administración exclusiva de tapentadol en un grupo en contraste con un grupo control al que se le administró analgesia estándar con fármacos tradicionales (antiinflamatorios no esteroideos). El muestreo fue no probabilístico (secuencial), con asignación aleatoria a cada grupo. La pupilometría por medio de AlgiScan y la valoración de escalas visuales análogas (EVA) se realizaron por parte de un investigador cegado a la intervención analgésica en curso. Se ingresó un total de 40 pacientes al ensayo, 19 al grupo al que se le administró tapentadol y 21 al grupo de analgesia estándar.

Las edades, pesos y tallas entre grupos (los que hubiesen recibido tapentadol contra otra analgesia) fueron comparados con la prueba no paramétrica de suma de rangos, U de Mann-Whitney-Wilcoxon, no encontrándose diferencias estadísticamente significativas entre los mismos. La representación de ambos sexos en los dos grupos fue evaluada con los instrumentos $\chi^{2}$ de Pearson (prueba paramétrica) y prueba exacta de Fisher (prueba no paramétrica), no encontrándose diferencia significativa entre grupos. Para la comparación de escalas de gravedad entre grupos (Charlson, SOFA y APACHE II) se utilizaron pruebas de comparación de medias (t de Student o análisis de varianzas, ANOVA o ANDEVA) apropiadas para el caso. En ninguna de las comparaciones se encontraron diferencias estadísticamente significativas (Cuadro 3).

\section{RESULTADOS}

Se constató una mortalidad similar entre grupos, con cinco fallecimientos en el grupo de tapentadol (26\%) contra cuatro fallecimientos en el grupo de otras analgesias (19\%), siendo la diferencia estadísticamente no significativa al ser analizada por medio de $\chi^{2}$ de Pearson y prueba exacta de Fisher (Cuadro 4). 


\begin{tabular}{|c|c|c|c|}
\hline & $\begin{array}{c}\text { Grupo } \\
\text { "tapentadol" } \\
(n=19)\end{array}$ & $\begin{array}{l}\text { Grupo "otra } \\
\text { analgesia" } \\
(n=21)\end{array}$ & $p$ \\
\hline Hombres & 9 & 11 & NS \\
\hline Mujeres & 10 & 10 & NS \\
\hline Edad & 70 años & 74 años & NS \\
\hline Peso & 69 kg & 70 kg & NS \\
\hline Talla & $1.65 \mathrm{~m}$ & $1.63 \mathrm{~m}$ & NS \\
\hline $\begin{array}{l}\text { APACHE II } \\
\text { (puntaje) }\end{array}$ & 21 & 23 & NS \\
\hline SOFA (puntaje) & 5 & 4 & NS \\
\hline $\begin{array}{l}\text { Charlson } \\
\text { (puntaje) }\end{array}$ & 1 & 2 & NS \\
\hline
\end{tabular}

Total de pacientes $=40$. Variables continuas reportados en medianas. NS = diferencia no significativa $(p>0.05)$. Se empleó el test estadístico $U$ de Mann-Whitney-Wilcoxon para muestras no distribuidas de manera normal. APACHE II = Acute Physiology and Chronic Health Evaluation. SOFA = Sequential Organ Failure Assessment. Charlson = índice de comorbilidades de Charlson.

Al comparar entre grupos el resultado numérico obtenido por AlgiScan en las instancias ya mencionadas, se encontró una diferencia estadísticamente significativa ( $p<$ 0.001), con una media de 11 (grupo de tapentadol) contra una media de 19 (grupo de otras analgesias), siendo clara la presencia de calificaciones de dolor menores en el grupo de tapentadol.

La evaluación por escala visual análoga (EVA) registró sólo una instancia de dolor en el grupo de tapentadol (5.3\%) contra 20 instancias (95.2\%) con la escala de EVA positiva en el grupo de otras analgesias, con significancia estadística $(p<0.001)$ según la prueba exacta de Fisher.

\section{DISCUSIÓN}

En México no existen estudios documentados en cuanto al diagnóstico y manejo del dolor en las UTI, pero el problema debe ser semejante a lo reportado a nivel internacional, lo cual requiere poner en alerta a las clínicas del dolor de los hospitales y a los médicos encargados del cuidado y manejo de pacientes críticamente enfermos. Las medidas no farmacológicas usualmente no se consideran en la atención del paciente grave, pero son fundamentales debido a que éste no se puede comunicar, por sus condiciones y por múltiples procedimientos a los que es sometido. Las medidas no farmacológicas que el intensivista debe procurar son: posición confortable, rascarse, deglutir, temperatura

\begin{tabular}{|c|c|c|c|}
\hline & $\begin{array}{c}\text { Grupo } \\
\text { "tapentadol" } \\
(\mathrm{n}=19)\end{array}$ & $\begin{array}{c}\text { Grupo "otra } \\
\text { analgesia" } \\
(\mathrm{n}=21)\end{array}$ & $\mathrm{p}$ \\
\hline Mortalidad & $5(26 \%)$ & 4 (19\%) & NS \\
\hline $\begin{array}{l}\text { AlgiScan } \\
\text { (puntaje) }\end{array}$ & 11 & 19 & $<0.001$ \\
\hline $\begin{array}{l}\text { EVA } \\
\text { RASS }\end{array}$ & $1(5.3 \%)$ & $20(95.2 \%)$ & $<0.001$ \\
\hline $\begin{array}{l}\text { Despertar } \\
\text { imposible }\end{array}$ & 0 & $1(4 \%)$ & \\
\hline $\begin{array}{l}\text { Sedación } \\
\text { profunda }\end{array}$ & 0 & 9 (42\%) & $<0.001$ \\
\hline $\begin{array}{l}\text { Sedación } \\
\text { moderada }\end{array}$ & $6(31 \%)$ & 11 (52\%) & \\
\hline $\begin{array}{l}\text { Sedación } \\
\text { ligera }\end{array}$ & 13 (68\%) & 0 & \\
\hline
\end{tabular}

Total de pacientes $=40$. Variables continuas $u$ ordinales reportados en medianas. NS = diferencia no significativa $(p$ $>0.05)$. EVA = Escala visual análoga del dolor, reportada en eventos compatibles con dolor. RASS $=$ Richmond AgitationSedation Scale. Se emplearon las pruebas estadísticas de $\chi^{2}$ de Pearson y prueba exacta de Fisher para comparación de porcentajes. Se empleó el test estadístico U de MannWhitney-Wilcoxon para muestras no distribuidas de manera normal.

ambiente adecuada, silencio, respetar la iluminación y la oscuridad de acuerdo con el ciclo de sueño-vigilia, música ambiental, comunicación verbal con el enfermo, compañía de familiares... Todas las medidas anteriores han demostrado que incrementan el confort, disminuyen la ansiedad, modulan el estado hiperadrenérgico e incrementan la confianza y el bienestar del enfermo. ${ }^{15}$

Soliman y cols. reportaron que en un estudio multicéntrico en Europa, donde se incluyeron 16 países, las tendencias del manejo del dolor en el paciente grave tenían una frecuencia de 20 a $80 \%$, lo que corrobora que habitualmente el dolor no se diagnostica en el paciente grave, por lo tanto no se trata. ${ }^{16}$

En la actualidad no hay un analgésico ideal para el paciente en terapia intensiva. Sin embargo, se eligió tapentadol por ciertas características que lo hacen benéfico como coadyuvante para tratar el dolor en el paciente crítico, baja unión a proteínas plasmáticas; no conduce a periodos de apnea y/o sedación, y al ser un opioide semisintético se usa al suspender infusiones de narcóticos, como equivalencia y bajo nivel adictivo. Se generó la duda de administrarlo en aquellos pacientes que se encuentran por más de 48 horas con infusión de sedación por opioides, en tiempo prolon- 
gado, y como destete para los que desarrollan síndrome de abstinencia por opioides; en este estudio se confirmó por videopupilómetro y por escala subjetiva EVA que el uso de tapentadol disminuyó, de manera segura y eficaz, el dolor en el paciente en terapia intensiva.

Diferentes estudios han mostrado alta prevalencia de dolor en el enfermo grave. Uno de los estudios epidemiológicos más grandes que investiga la incidencia del dolor en pacientes hospitalizados es el denominado SUPPORT (Study to Understand Prognoses and Preferences for Outcomes and Risk of Treatment). ${ }^{17}$

En otras palabras, la experiencia de dolor se puede guardar en la memoria, en la corteza somatosensorial, causando hipersensibilidad ante un estímulo previo poco doloroso; el dolor puede surgir y continuar por cierto tiempo. El manejo del dolor de manera agresiva puede hacer que el dolor permanezca, especialmente si tal manejo es capaz de disminuir el riesgo de cambios neurológicos que acompañan la persistencia del dolor. ${ }^{18}$

El impacto de la analgesia insuficiente se evidenció por el hecho de que los pacientes informaron que el dolor afecta las capacidades fisiológicas como toser, o inspirar profundamente. La percepción del dolor en el hospital y en casa (por los cuidadores) es subjetiva, debido a que los pacientes no informan del dolor a pesar de percibirlo, por miedo a efectos secundarios, al aumento de dosis de analgésicos o por temor a la adicción a opiáceos, conceptos erróneos por parte del familiar o del propio paciente. ${ }^{19}$

La tendencia clínica actual es proporcionar un nivel más ligero de sedación, menos días, y la interrupción diaria, debido a que el uso continuo de infusiones para sedación y analgesia presenta riesgo de neuroadaptación y dependencia fisiológica, y puede desarrollar síntomas de abstinencia. Se debe protocolizar en cada UTI la sedación deseada, siendo esencial que todos los miembros de la UTI sean conscientes del plan de analgesia, durante la sedación y el posterior retiro de ésta, además de adherirse al protocolo establecido y estar conscientes del potencial de deficiencias para ajustes apropiados de manera individualizada. ${ }^{20}$

\section{REFERENCIAS}

1. www.idmed.fr. Manual NeuroLight AlgiScan. Versión 1.15 A5 - SP. 2013; 1-36.
2. Joffe AM, Hallman M, Gélinas C, Herr DL, Puntillo K. Evaluation and treatment of pain in critically ill adults. Semin Respir Crit Care Med. 2013; 34 (2): 189-200.

3. Mauro ODDO, Park RP, Lane M. Sedation and analgesia. PACT (patient-Centred Acute Care Training). Update 2010; 220-224.

4. Sessler CN, Grap MJ, Ramsay MA. Evaluating and monitoring analgesia and sedation in the intensive care unit. Crit Care. 2008; 12 Suppl 3: S2.

5. Barr J, Pandharipande PP. The pain, agitation, and delirium care bundle: synergistic benefits of implementing the 2013 Pain, Agitation, and Delirium Guidelines in an integrated and interdisciplinary fashion. Crit Care Med. 2013; 41 (9 Suppl 1): S99-S115.

6. Stites M. Observational pain scales in critically ill adults. Crit Care Nurse. 2013; 33 (3): 68-78.

7. Barr J, Fraser GL, Puntillo K, Ely EW, Gélinas C, Dasta JF et al. Clinical practice guidelines for the management of pain, agitation, and delirium in adult patients in the intensive care unit. Crit Care Med. 2013; 41 (1): 263-306.

8. Chanques G, Pohlman A, Kress JP, Molinari N, de Jong A, Jaber S et al. Psychometric comparison of three behavioural scales for the assessment of pain in critically ill patients unable to self-report. Crit Care. 2014; 18 (5): R160.

9. Battle $\mathrm{CE}$, Lovett $\mathrm{S}$, Hutchings $\mathrm{H}$. Chronic pain in survivors of critical illness: a retrospective analysis of incidence and risk factors. Crit Care. 2013; 17 (3): R101.

10. de Jong A, Molinari N, de Lattre S, Gniadek C, Carr J, Conseil M et al. Decreasing severe pain and serious adverse events while moving intensive care unit patients: a prospective interventional study (the NURSE-DO project). Crit Care. 2013; 17 (2): R74.

11. Mendis V. Role of tapentadol in pain management. Br J Hosp Med (Lond). 2012; 73 (3): 143-147.

12. Candiotti KA, Gitlin MC. Review of the effect of opioid-related side effects on the undertreatment of moderate to severe chronic noncancer pain: tapentadol, a step toward a solution? Curr Med Res Opin. 2010; 26 (7): 1677-1684.

13. Ahlbeck K. Opioids: a two-faced Janus. Curr Med Res Opin. 2011; 27 (2): 439-448.

14. Gudin JA. Assessment of extended-release opioid analgesics for the treatment of chronic pain. J Pain Palliat Care Pharmacother. 2013; 27 (1): 49-61.

15. Carrillo R. Dolor en el paciente críticamente enfermo. Dolor por especialidades. 2a edición. Corinter; 2008. Tomo I: 601-619.

16. Soliman HM, Mélot C, Vincent JL. Sedative and analgesic practice in the intensive care unit: the results of a European survey. Br J Anaesth. 2001; 87 (2): 186-192.

17. Murphy P, Kreling B, Kathryn E, Stevens M, Lynn J, Dulac J. Description of the SUPPORT intervention. Study to understand prognoses and preferences for outcomes and risks of treatments. J Am Geriatr Soc. 2000; 48 (5 Suppl): S154-S161.

18. Puntillo KA, Max A, Chaize M, Chanques G, Azoulay E. Patient recollection of ICU procedural pain and post ICU burden: the memory study. Crit Care Med. 2016; 44 (11): 1988-1995.

19. Benzon H, Rathmell J, Wu CL, Turk D, Argoff C, Hurley R. Practical management of pain. Practical Management in the critically ill patient. 5th ed. Mosby; 2014. pp. 1015-1022.

20. Benzon H, Raja SN, Fishman S, Liu S, Cohen SP. Essentials of pain medicine. Pain control in the critically ill patients. 3rd ed. Saunders; 2011. pp. 253-260. 